ARTÍ́CULO DE REVISIÓN

\title{
Consenso de Prediabetes. Documento de posición de la Asociación Latinoamericana de Diabetes (ALAD)
}

Juan Rosas-Saucedo ${ }^{1 *}$, A. Enrique Caballero², Griselda Brito-Córdova ${ }^{3}$, Hernán García ${ }^{4}$, José Costa-Gil ${ }^{5}$, Ruy Lyra ${ }^{6}$ y Juan Rosas-Guzmán ${ }^{1}$

${ }^{1}$ Instituto de Diabetes de Celaya, AC. Celaya, México; ${ }^{2}$ Harvard Medical School, Boston, EE.UU.; ${ }^{3}$ nstituto Nacional de Ciencias Médicas y Nutrición Salvador Zubirán, Delegación Tlalpan, Ciudad de México, México; ${ }^{4}$ Pontificia Universidad Católica, Santiago de Chile, Chile; ${ }^{5}$ Universidad Favaloro, Ciudad Autónoma de Buenos Aires, Argentina; ${ }^{6}$ Hospital das Clinicas, Pernambuco Federal University, Pernambuco, Recife, Brasil

\section{RESUMEN}

La diabetes mellitus es un problema de salud pública en constante ascenso en la mayor parte del mundo. Latino-América (LA) presenta una prevalencia elevada de diabetes mellitus tipo 2 (DM2), en la población mayor de 20 años y fluctúa entre 8 y $10 \%$. Esta prevalencia se ha incrementado acorde con el patrón epidemiológico mundial; sin embargo, es particularmente elevada en LA por las características genéticas de la población, hábitos de alimentación inadecuados y sedentarismo que, vinculados con el síndrome metabólico, han encontrado un ambiente favorable para su expresión con nuestro estilo de vida actual. La DM2 es una enfermedad crónica, degenerativa, progresiva pero controlable. En la actualidad se conocen algunos mecanismos fisiopatológicos que se manifiestan en diferentes etapas de su historia natural. Dentro de este marco de referencia se desprende que la gran mayoría de los casos

\section{ABSTRACT}

Diabetes mellitus is a growing public health problem in most parts of the world. Latin America (LA) has a high prevalence of type 2 diabetes mellitus (DM2), in the population over 20 years and fluctuates between 8 and $10 \%$. This prevalence has increased according to the global epidemiological pattern; how ever, it is particularly elevated in LA because of the genetic characteristics of the popula tion, inadequate eating habits and sedentary lifestyle, which, associated to the metabolic syndrome, have found a favorable environ ment for their expression with our current lifestyle. DM2 is a chronic, degenerative, pro gressive but controllable disease. Nowadays, we know that some pathophysiological mechanisms do manifest in different stages of its natural history. Within this framework, it can be concluded that the wide majority of $\mathrm{DM} 2$ cases occur in individuals who, during a variable period of years, possibly decades,
* Juan Rosas-Saucedo

E-mail: drjrosas@gmail.com
Fecha de recepción: 31-08-2017

Fecha de aceptación: 11-09-2017 DOI: 10.24875/ALAD.17000307 
de $\mathrm{DM} 2$ se presentan en individuos que, durante un período variable de años, posiblemente décadas, han cursado con alteraciones metabólicas que preceden y acompañan al estado de hiperglucemia persistente. En LA, la $\mathrm{DM} 2$ tiene un gran impacto en la economía del sistema de salud. Los pacientes con DM2 tienen hospitalizaciones más prolongadas y es la segunda causa de mortalidad en pacientes hospitalizados.

Palabras clave: Diabetes. Prediabetes. Latinoamérica. Glucosa alterada de ayuno. Intolerancia a la glucosa. have developed metabolic alterations that precede and accompany persistent hyperglycemia. In LA, DM2 has a major impact on the health system economy. Patients with $\mathrm{DM}_{2}$ have longer hospitalizations and are the second main cause of mortality in hospitalized patients. (Rev ALAD. 2017;7:184-202) Corresponding author: Juan Rosas-Saucedo, drjrosas@gmail.com

Key words: Diabetes. Prediabetes. Latin-America Impaired fasting glucose. Impaired glucose tolerance.

\section{INTRODUCCIÓN}

La diabetes mellitus es un problema de salud pública en constante ascenso en la mayor parte del mundo. Latinoamérica ( $L A)$ presenta una prevalencia elevada de diabetes mellitus tipo 2 (DM2) en la población mayor de 20 años y fluctúa entre el 8 y el $10 \%$. Esta prevalencia se ha incrementado acorde con el patrón epidemiológico mundial; sin embargo, es particularmente elevada en LA por las características genéticas de la población, hábitos de alimentación inadecuados y sedentarismo que, vinculados con el síndrome metabólico, han encontrado un ambiente favorable para su expresión con nuestro estilo de vida actual.

La DM2 es una enfermedad crónica, degenerativa y progresiva, pero controlable. En la actualidad se conocen algunos mecanismos fisiopatológicos que se manifiestan en diferentes etapas de su historia natural. Dentro de este marco de referencia se desprende que la gran mayoría de los casos de DM2 se presentan en individuos que durante un periodo variable de años, posiblemente décadas, han

cursado con alteraciones metabólicas que precềden y acompañan al estado de hiperglucemia per̂ sistente.

En LA, la DM2 tiene un gran impacto en la economía del sistema de salud. Los pacientes con $\mathrm{DM} 2$ tienè hospitalizaciones más prolongadas y es la segunda causa de mortalidad en pacientes hospitalizados. Desde una perspectiva económica, todos pagamos: los pacientes, sus familias, el gasto asociado con la atención médica mediante el pago de impuestošs, los días laborales no trabajados, pérdida de empleô, etc. Aún más, en EE.UU. el 70\% del presupuesto dišsponible para el tratamiento de pacientes con diabè tes se emplea en tan solo el $15 \%$ de los pacientes; habitualmente aquellos con complicaciones de enfermedad.

Por otra parte, la DM2 es la causa de por lo menós el $40 \%$ de los casos de insuficiencia renal crónica tratados con diálisis peritoneal o hemodiálisis. Se estima que en 2005 el costo total de la atención de pacientes con DM2 en LA fue de 317 millones de dólares. Estas cifras reflejan la importancia de desarrollar 
estrategias efectivas de prevención, detección temprana, atención oportuna y control.

Dentro de la historia natural de la enfermedad se ha señalado un estado metabólico previo que no corresponde a diabetes pero que tampoco se ubica dentro de la normalidad, es decir, se trata de un estado intermedio que se ha definido como prediabetes. La importancia de este conocimiento se ha puesto de manifiesto porque en una decena de estudios se ha demostrado que al identificar e intervenir en el estilo de vida a estos pacientes es posible evitar su progresión a diabetes hasta en un $58 \%$ de los casos. Se estima que la prediabetes conlleva una disminución de la reserva pancreática y que, al momento de manifestarse el estado diabético, la reserva está reducida en por lo menos un 50\%. En teoría, al intervenir en los pacientes en estado de prediabetes se podría evitar el deterioro progresivo de las células beta o, por lo menos, desacelerarlo. Es razonable anticipar que la detección y tratamiento de la prediabetes sea una estrategia eficiente para lidiar con la epidemia de DM2.

Para numerosos individuos el diagnóstico de DM2 es un suceso tardío, relativo al entorno global de su salud, y es frecuente que coexistan e incluso le antecedan otros factores de daño vascular que forman parte del síndrome metabólico, como la dislipidemia, la resistencia a la insulina, la hipertensión arterial e inclusive que haya presentado alguna complicación vascular antes del diagnóstico de DM2. Los argumentos mencionados constituyen la justificación para elaborar criterios de detección y tratamiento de prediabetes.

En personas menores de 20 años de edad, se ha observado un aumento constante en los casos de DM2 durante las últimas 2-3 décadas, pasando de ser de una condición clínica previamente desconocida en edades pediátricas a ser cada vez más frecuente en niños y adolescentes, tanto en países desarrollados como en vías de desarrollo, como los nuestros.

\section{La prediabetes como problema epidemiológico en LA}

Con el crecimiento de la población, la modificación en los estilos de vida y otros factores de riesgo, enfermedades como la DM2 y los eventos cardiovasculares se han incrementado en forma paralelă ambas entidades pueden presentarse en forma in dependiente o interrelacionadas. Se considera a ta $\mathrm{DM} 2$ como un problema de salud pública dada șu alta prevalencia y su incremento acelerado en lợ últimos 20 años; la Organización Mundial de la Sălud (OMS) ha señalado que en países de LA este incremento puede ser hasta del $160 \%$ en los próxìmos 25 años. Con el apoyo de estudios epidemiolögicos y el conocimiento de que es posible identificằr a la DM2 en etapa asintomática (debido a que la hiperglucemia es el factor determinante de las complicaciones microvasculares y uno de los factoreso marcadores de riesgo de complicaciones macrovaśculares), se han desarrollado estrategias de detección temprana con procedimientos diagnósticôs económicos, sencillos y sensibles, con la finalidăd de tomar medidas de prevención oportuna.

En EE.UU., en el NHANES III (National Health and Nutrition Examination Survey III) se encontró qựe el $22.6 \%$ de adultos de 45 a 74 años con sobrepeso tienen prediabetes. De ellos, el $51.2 \%$ tenían soto tolerancia a la glucosa alterada (TGA), el 23.5\% la glucosa en ayuno alterada (GAA) y el $25.2 \%$, GAA $y$ TGA combinadas.

Estas cifras son similares a las reportadas en otros países como Suecia (22\%), Australia (23.7\%) Singapur (23\%) y Corea, lo que señala una prevalencia mundial 
de prediabetes que varía entre el 15 y el $25 \%$. De acuerdo con la Federación Internacional de Diabetes, en el 2013 alrededor de 382 millones de personas tenían diabetes, de las cuales el $80 \%$ vivían en países de ingresos medios y bajos. Para ese mismo año, la región de América Central y América del Sur concentró 24.1 millones de personas con diabetes y se estima que para el 2035 esta cifra aumente en un $59.8 \%$.

Un punto de particular interés en nuestra población es que adultos jóvenes latinos con historia familiar de diabetes, aun teniendo cifras normales de glucosa, ya muestran disfunción endotelial e inflamación vascular significativa. Estas mismas alteraciones se han demostrado desde la infancia y la adolescencia en personas con sobrepeso.

La prevalencia nacional de diabetes en LA varía notablemente entre países, siendo Perú el que registra la cifra más baja (4.3\%) y Puerto Rico la más alta (15.4\%). En todo el mundo, Brasil y México se ubican en el cuarto y el sexto lugar en la lista de países con el mayor número de personas con diabetes entre los 20 y 79 años de edad. En la Encuesta Nacional de Salud y Nutrición (ENSANUT) 2016 la prevalencia de diabetes en México se reportó en un 9.4\%, tomando en consideración los casos conocidos con diabetes en el momento de la encuesta; próximamente se dará a conocer la prevalencia total al considerar los casos incidentes.

En México, los datos de la ENSANUT 2006 muestran una prevalencia de $\mathrm{DM} 2$ en adultos mayores de 20 años de edad del 10.7\%, presentándose la GAA en el $12.7 \%$ de los casos. Si se aplica la nueva recomendación de la American Diabetes Association (ADA) de noviembre del 2004, de bajar el punto de corte de glucosa normal a menos de $100 \mathrm{mg} / \mathrm{dl}$, la prevalencia de la GAA se incrementa a $20.1 \%$, lo que representa 16 millones de mexicanos mayores de
20 años de edad en alto riesgo de progresar a diabetes.

De hecho, la prevalencia de DM2 tiende a seguir aumentando. La Secretaría de Salud de México informó de que la prevalencia de la diabetes pasó de $8.2 \%$ en el año 2000 a $10.7 \%$ en 2006 . En datos dề año 2005 , la mortalidad en mujeres mexicanas fừe del 66.6 y en hombres del 56.7 por 100,000 habitañ tes, ubicándose como causa número uno de muertê.

En jóvenes de 12-19 años, la NHANES III arrojó uñăa prevalencia de todos los tipos de DM del 4.1 pö̀r 1,000 jóvenes, de los cuales aproximadamente ự 30\% padecen $\mathrm{DM}_{2}$; prevalencia que continúa aŭ mentando. Esta prevalencia en EE.UU. es mayor en grupos étnicos como los indios nativos americanos, afroamericanos e hispanos.

En datos latinoamericanos, la encuesta Nacional de Salud de Chile realizada en 2010 registró una prevă lencia de la DM2 del $0.4 \%$ entre los 15 y los 24 añoș, con marcado predominio femenino en el grupo más joven (4:1).

\section{Definición}

En 1965, el comité de expertos de la OMS utilizó è término de prediabetes aplicándolo en forma rè trospectiva a individuos con diabetes diagnostică da, pero hay algunas publicaciones anteriores en lös cuales ya se utiliza este término (p. ej., en la revista Diabetes de julio-agosto de 1962, WP Jackson escríbió el artículo That expression; prediabetes).

El modelo de estudio e investigación incluía la etapa de prediabetes en las fases evolutivas de la enfermedad, considerándola la fase más temprana y que solo se podía sospechar, pero no diagnosticar. Incluía alteraciones genéticas que hacían al individuo 
susceptible de desarrollar DM2 a lo largo del tiempo, pero que no presentaban alteraciones demostrables en las pruebas diagnósticas. En este modelo se incluían otras categorías no utilizadas actualmente como la diabetes subclínica y la diabetes latente.

En 1979, el NDDG (National Diabetes Data Group de EE.UU.), en colaboración con el Comité de Expertos de la OMS, propusieron una clasificación en la que se definió la categoría clínica de intolerancia a la glucosa y las categorías estadísticas de «anormalidad previa» $\mathrm{y}$ «anormalidad potencial» a la tolerancia a la glucosa. Pese a que reconocían que estos diagnósticos identificaban individuos con alto riesgo a desarrollar diabetes, no usaron el término de prediabetes.

Pero no es hasta el 27 de marzo del 2003 cuando la ADA, con base en los resultados del Diabetes Prevention Program (DPP), toma una posición y propone una definición de la prediabetes:

«Es un estado que precede al diagnóstico de diabetes tipo 2. Esta condición es común, está en aumento epidemiológico y se caracteriza por elevación en la concentración de glucosa en sangre más allá de los niveles normales sin alcanzar los valores diagnósticos de diabetes. Se puede identificar mediante una prueba de tolerancia oral a la glucosa (tolerancia a la glucosa alterada, TGA) o mediante la glucemia en ayunas (glucosa alterada de ayuno, GAA). La mayoría de las personas con cualquiera de las dos condiciones desarrollará diabetes manifiesta dentro de un periodo de 10 años.»

Tanto la GAA como la TGA están íntimamente relacionadas con el síndrome metabólico y no solo indican alto riesgo para el desarrollo de diabetes manifiesta, también, y en forma similar al síndrome metabólico, indican riesgo de enfermedad vascular ateroesclerótica.

\section{Diagnóstico}

En función de la definición presentada previamente, el diagnóstico de la prediabetes se establece exclusivamente con determinación de la concentración de glucosa en plasma. Los valores específicồs recomendados son:

- TGA: Glucosa plasmática entre 140 y 199 mg/d (7.8 a $11 \mathrm{mmol} / \mathrm{l}$ ), medidos $2 \mathrm{~h}$ después de una carga oral de $75 \mathrm{~g}$ de glucosa anhidra diluida en $300 \mathrm{ml}$ de agua, debiéndose ingerir en menos de 5 minutos.

- GAA: glucosa plasmática después de un ayuno de $8 \mathrm{~h}$ y que resulte entre 100 y $125 \mathrm{mg} / \mathrm{dl}$, (6.1 $6.9 \mathrm{mmol} / \mathrm{l})$, de acuerdo a la recomendación püblicada por la ADA en 2003.

Los criterios y puntos de corte recomendados actualmente para diagnóstico de normalidad de tolếrancia a los hidratos de carbono, GAA, TGA, combinación de GAA + TGA y diabetes manifiesta se ilustran en la tabla 1 y figura 1.

Varios estudios han indicado discordancia entre lös diagnósticos de GAA y TGA. Los dos ejemplos mås

TABLA 1. Criterios diagnósticos de normalidad, Prediabetes y diabetes. GAA, glucosa en ayuno alterada; TGA, tolerancia a la glucosa alterada

\begin{tabular}{|c|c|c|}
\hline \multirow{2}{*}{$\begin{array}{l}\text { Diagnóstico } \\
\text { metabólico }\end{array}$} & \multicolumn{2}{|c|}{ Glucosa plasmática (mg/dl) } \\
\hline & Ayuno & $\begin{array}{c}2 \text { h poscarga } \\
\text { de glucosa }\end{array}$ \\
\hline Normal & $<100$ & $<140$ \\
\hline GAA & $100-125$ & $<140$ \\
\hline TGA & $<100$ & $140-199$ \\
\hline GAA + TGA & $100-125$ & $140-199$ \\
\hline Diabetes & $\geq 126$ & $\geq 200$ \\
\hline
\end{tabular}




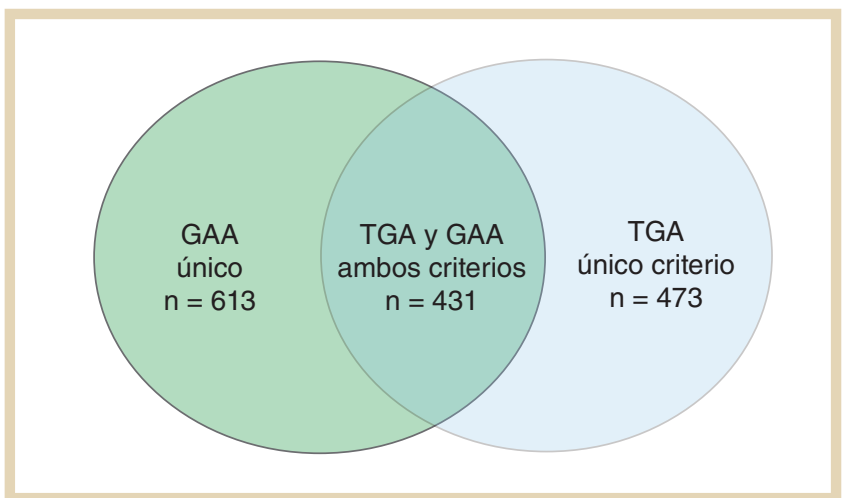

Figura 1. Interacción entre tolerancia a la glucosa alterada (TGA) y glucosa alterada en ayuno (GAA). FPG, glucosa plasmática en ayunas; 2-hPG, glucosa plasmática a las $2 \mathrm{~h}$.

Tomado de: The DECODE Study Group, the European Diabetes Epidemiology Group. Is the current definition for diabetes relevant to mortality risk from all causes and cardiovascular and non-cardiovascular diseases? Diabetes Care. 2003;26:688-96.

significativos son el estudio DECODE (Diabetes epidemiology: collaborative analysis of diagnostic criteria in Europe) y la NHANES III. En el DECODE, el $28 \%$ de los participantes con alteraciones en la tolerancia a los hidratos de carbono tienen las dos alteraciones (GAA + TGA), un 40\% encajan en el grupo de GAA y un $31 \%$ en el grupo de TGA. Por su parte, la NHANES III estudió sujetos de 40 a 74 años de edad sin diagnóstico previo de anormalidad en metabolismo de hidratos de carbono. De los sujetos investigados en quienes se demostró alguna alteración del metabolismo de los hidratos de carbono, un $44 \%$ tenían alteraciones combinadas (GAA + TGA), un $14 \%$ presentaron GAA aislada y un $41 \%$, TGA.

Algunos datos de investigación sugieren que la GAA y la TGA son categorías diferentes de tolerancia a la glucosa con fisiopatologías diversas. Los individuos con GAA tienen resistencia a la insulina más acentuada, mientras que la TGA parece ser secundaria a deficiencia de secreción de insulina posingesta de glucosa (o alimentos). El riesgo de diabetes aumenta cuando ambas categorías de tolerancia a glucosa alterada coexisten. No sorpresivamente, la concentración de glucosa en el periodo posingestión de glucosa se eleva en forma más acentuada en sujetos que presentan las dos alteraciones que en aqueIlos que solo muestran una de ellas.

La interpretación de los datos disponibles se com plica aún más, ya que ambos estados metabólicờs representan un estado altamente dinámico.

La GAA puede:

- Revertir a un estado de glucemia en ayuno nor mal.

- Progresar a TGA o DM2.

- Mantenerse como GAA.

La TGA puede:

- Revertir a tolerancia a la glucosa normal.

- Progresar a síndrome de resistencia a la insulină.

- Progresar a DM2.

- Mantenerse como TGA.

Desafortunadamente, y debido a cambios ambien tales negativos (obesidad y sedentarismo), la evolüción más probable en ambos casos es hacia el detérioro metabólico con aparición de diabetếs manifiesta en una muy alta proporción de las peŕsonas con GAA o TGA. Este es el reto fundamentă que LA enfrenta en nuestro tiempo. La evolución de estos estados metabólicos ha sido documentada en varios estudios prospectivos:

En el estudio Singapur de 2002, los investigadores documentaron que después de ocho años de 
observación, el 14\% de sujetos inicialmente tolerantes a la glucosa evolucionaron hacia la TGA y el $4.3 \%$ a diabetes. Del total de sujetos que habían progresado a TGA, un $41 \%$ revirtió a tolerancia normal, un 23\% permaneció en TGA y un 35.1\% progresó a diabetes manifiesta.

En Cuba, Amador Perichetal documentó la evolución de 114 sujetos con TGA por espacio de 18 años, al cabo de los cuales el $78 \%$ permanecían vivos. El $54 \%$ evolucionó a diabetes manifiesta, el 23\% revirtieron a tolerancia normal y el resto mantuvo TGA. El estudio HOORN incluyó a 1,428 individuos, que fueron evaluados durante seis años. De los casos incidentes de diabetes, un $82 \%$ presentaron intolerancia a hidratos de carbono antes de manifestar diabetes (40\% TGA, 42\% GAA).

Aunque no tenemos datos definitivos para definir si estos dos estados metabólicos son diferentes o representan fases evolutivas de un proceso similar, un argumento importante por determinar es si la GAA y la TGA son procesos diferentes o estas categorías han sido creadas artificialmente por los puntos de corte escogidos. Con respecto a lo anterior, los datos recientemente publicados por investigadores del Instituto Nacional de Ciencias Médicas y Nutrición Salvador Zubirán de México (INCMNSZ) son muy relevantes. Los autores de este proyecto investigaron la validez del punto de corte de glucemia plasmática en ayuno propuesto por la ADA y la OMS (100-125 mg/dl) como valor predictivo de intolerancia a los hidratos de carbono en la curva de tolerancia oral a la glucosa. Los datos de este estudio y el análisis estadístico apoyan un punto de corte más bajo de la GAA (alrededor de $95 \mathrm{mg} / \mathrm{ml}$ ). Este punto de corte tiene mayor poder predictivo de la TGA que el valor actualmente recomendado por la ADA y la OMS. La ventaja potencial de disminuir el punto de corte para diagnóstico de la GAA radica en varios aspectos: 1) convergencia de diagnósticos de TGA y la GAA, 2) mejoría del cribado y la vigilancia epidemiológica, ya que la implementación en la población general de la GAA es más económica que la curva de tolerancia a la glucosa.

Pese a la validez interna de este estudio, hay algùnos aspectos que necesitan ser definidos antes de que se pueda hacer una recomendación oficial de cambio. El estudio se llevó a cabo en una sola instị tución y la muestra es reducida. Los participantês tienen un sesgo de selección, ya que el INCMNSZ es reconocido como un centro de tercer nivel en Mĕ́xico con alta concentración de pacientes con diabê tes. La población de pacientes del INCMNSZ no ề representativa de la población general de LA. Pör lo tanto, se necesitan estudios epidemiológicợs prospectivos para conferir validez externa a esta recomendación que incluyan las poblaciones sĭn sesgo de selección y representativas de LA. Desăfortunadamente, no existen estudios específicos de prevención de diabetes usando la glucosa plasmáțica en ayuno como criterio de selección.

Reconociendo esta discordancia entre la GAA y ta TGA, la ADA recomienda la GAA como la prueba preferida para la búsqueda del diagnóstico de anơmalidad en el metabolismo de hidratos de carbono. Otras organizaciones internacionales como la Eurŏ. pean Society of Cardiology y la European Associätion for the Study of Diabetes se inclinan más hacĩa la curva de tolerancia a la glucosa oral.

\section{Algoritmos de detección}

El uso de una encuesta para la estratificación de riesgo para prediabetes sería un abordaje sencillo, aplicable a la población general, que permitiría la detección de los individuos en riesgo que ameriten una evaluación bioquímica para determinar su tolerancia a la glucosa. Cuando se identifica por 
encuesta a los individuos en riesgo se pasa a una evaluación bioquímica de los mismos.

Los individuos con prediabetes pudieran clasificarse como: individuos con GAA, individuos con TGA o con ambas.

En una estrategia rentable de detección oportuna de casos de los individuos en riesgo se sugiere tomar en cuenta la suma de diversos factores que han demostrado incrementar el riesgo de disglucemia:

- Antecedente hereditario familiar.

- Sobrepeso u obesidad.

- Signos clínicos o bioquímicos de resistencia a la insulina (acantosis nigricans, hipertensión arterial, hipertrigliceridemia, colesterol HDL (lipoproteínas de alta densidad) bajo o síndrome de ovarios poliquísticos).

Para la identificación en población general de los individuos en riesgo de prediabetes, la Secretaría de Salud de México ha propuesto la aplicación de una encuesta y evaluación clínica con un instrumento que contiene diversos elementos. En la propuesta, la encuesta y el sistema de estratificación son diferentes para la población pediátrica y la adulta. El sistema de puntuación propuesto se presenta en las tablas 2 y 3 .

La propuesta recomienda que una puntuación mayor de 12 en la población pediátrica, y mayor de 9 en adultos sea seguida de escrutinio glucémico con determinación de glucosa en ayunas y 2 horas poscarga de glucosa oral (75 g de glucosa anhidra).

De acuerdo a los criterios de diagnóstico presentados previamente, se establece la presencia de prediabetes en cualquiera de los casos presentados en la tabla 4 .
TABLA 2. Sistema de detección de riesgo de prediabetes en México. Población pediátrica

\begin{tabular}{|c|c|c|c|}
\hline \multicolumn{4}{|c|}{ Sistema de puntuación para población pediátrica } \\
\hline & Valor & Puntos & \\
\hline \multirow{5}{*}{$\begin{array}{l}\text { Índice de masa } \\
\text { corporal (percentil) }\end{array}$} & $<85$ & 0 & F \\
\hline & $85 a<95$ & 1 & 9 \\
\hline & $95 a<97$ & 2 & 4 \\
\hline & 97 & 3 & z \\
\hline & Obesidad mórbida & 4 & $\frac{a f}{0}$ \\
\hline \multirow[t]{4}{*}{ Historia familiar DM2 } & Negativa & 0 & \\
\hline & Madre & 2 & 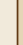 \\
\hline & Padre & 1 & $\bar{z}$ \\
\hline & Ambas & 2 & 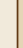 \\
\hline \multirow{3}{*}{$\begin{array}{l}\text { Signos de resistencia } \\
\text { a la insulina }\end{array}$} & Acantosis nigricans & 2 & \\
\hline & Ovario poliquístico & 4 & 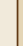 \\
\hline & $\begin{array}{r}\text { Hipertensión, } \\
\text { dislipidemia }\end{array}$ & 4 & \\
\hline
\end{tabular}

TABLA 3. Sistema de detección de riesgo de prediabetes en México. Población adulta

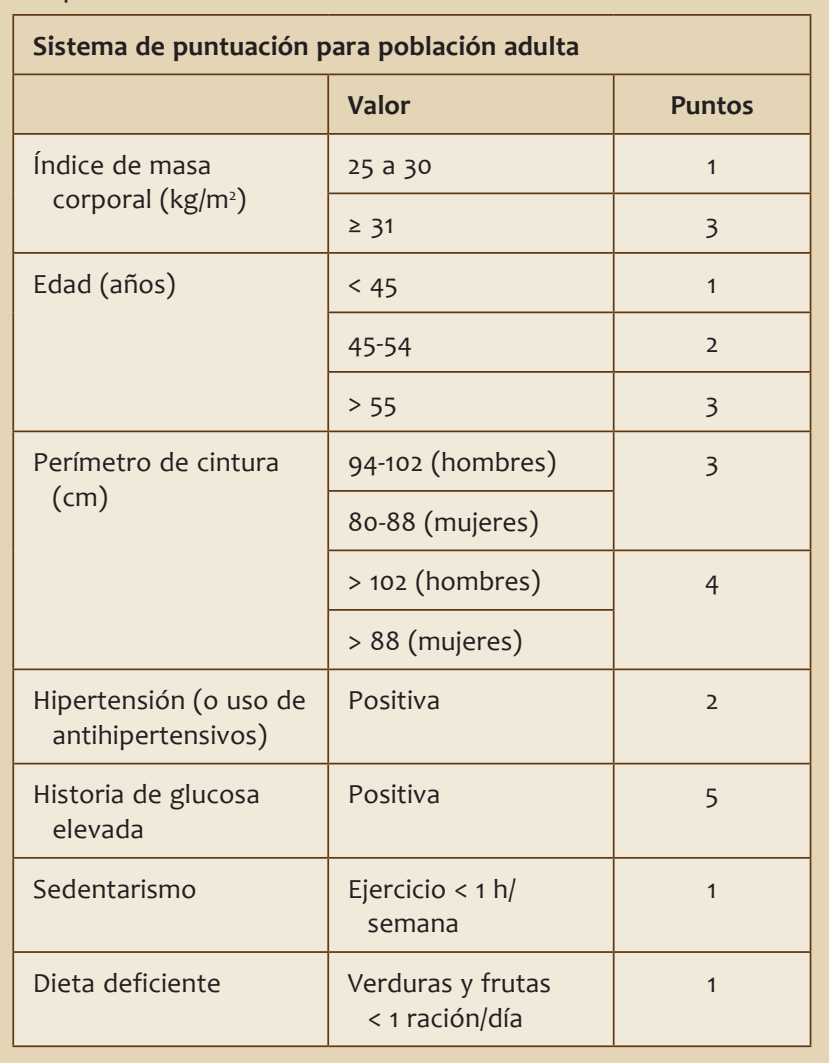


TABLA 4. Categorías de riesgo incrementado para diabetes (prediabetes)*. HbA1c: prueba de la hemoglobina glucosilada

\begin{tabular}{|l|}
\hline Glucemia en ayunas: $100-125$ mg/dl (GAA) \\
\hline Glucemia 2 h en una CTOG con 75 g entre: $140-199$ mg/dl (TGA) \\
\hline HbA1c: $5 \cdot 7-6.4 \%$ \\
\hline
\end{tabular}

*Para las tres pruebas, el riesgo es continuo.

El criterio de hemoglobina glucosilada (Tabla 4) debe tomarse con cautela y excluirla con fines diagnósticos si no se cuenta con laboratorios debidamente estandarizados para realizar dicha determinación.

Valores inferiores no establecen el diagnóstico, pero obligan al seguimiento anual con la misma prueba. Si bien este sistema es útil en población, a nivel individual los médicos pueden ampliar la cobertura de detección con la presencia de uno o más factores de riesgo señalados en la tabla 5 .

TABLA 5. Criterios de escrutinio de diabetes o prediabetes en adultos asintomáticos

1. Se debe considerar la prueba en adultos con sobrepeso u obesidad (IMC $\geq 25 \mathrm{~kg} / \mathrm{m}^{2} \mathrm{O} \geq 23$ en asiaticoamericanos) con uno o más de los siguientes factores de riesgo: $\mathrm{HbA} 1 \mathrm{c} \geq 5.7 \%$ (39 $\mathrm{mmol} / \mathrm{mol})$, TGA o GAA

Familiar de primer grado con diabetes

Alto riesgo racial/étnico (p. ej., afroamericanos, latinos, americanos nativos, asiaticoamericanos, isleños del Pacífico)

Mujeres con diagnóstico de diabetes mellitus gestacional Historia de enfermedad cardiovascular Hipertensión ( $\geq 140 / 90$ mmHg o en terapia para hipertensión) Colesterol $\mathrm{HDL}<35 \mathrm{mg} / \mathrm{dl}(0.90 \mathrm{mmol} / \mathrm{l}) \mathrm{y} / \mathrm{o}$ nivel de triglicéridos $>250 \mathrm{mg} / \mathrm{dL}(2.82 \mathrm{mmol} / \mathrm{l})$

Mujeres con síndrome de ovarios poliquístico Inactividad física

Otras condiciones clínicas asociadas con resistencia a la insulina (p. ej., obesidad severa, acantosis nigricans)

2. Para todos los pacientes, las pruebas deben hacerse antes de los 45 años

3. Si los resultados son normales, las pruebas deben repetirse al menos a los tres años, considerando pruebas más frecuentes dependiendo del resultado inicial ( $p$, ej., aquellos con prediabetes deben repetir las pruebas de laboratorio anualmente) y el riesgo

\section{Tratamiento de la prediabetes}

En los últimos diez años, varios ensayos clínicos han sido publicados con respecto al tratamiento de la prediabetes con la finalidad de investigar la efectividad de estos tratamientos en retardar o abrogăr la progresión de la prediabetes a diabetes manifies. ta. Resulta importante destacar que la prediabetè no es una enfermedad separada, sino que el desă rrollo de la DM2 es un proceso gradual que ocurre durante años. En forma general, los estudios mues. tran datos optimistas y permiten establecer que: 1 (1) cambios en el estilo de vida son altamente efectivơs en retardar la progresión de prediabetes a diabetề y 2) los agentes farmacológicos que aumentan la sensibilidad a la insulina (metformina, glitazonas) 0 que impiden la absorción de hidratos de carbono (acarbosa) también confieren un efecto de retardo en la progresión de prediabetes a diabetes. Pérdidas de peso moderadas se pueden traducir en prevención de la enfermedad en personas de alto ries go. Las personas con TGA que recibieron intervenciōn intensa en el estilo de vida en el DPP tuvieron una pérdida de peso de $5.6 \mathrm{~kg}$ en 2.8 años y un $58 \%$ rềducción del riesgo relativo de $\mathrm{DM} 2$.

En forma simplificada, el tratamiento de individuos con prediabetes incluye primordialmente el cambio en el estilo de vida, teniendo como metas la pérdida de peso y el aumento en el ejercicio físico cotidianồ. Si estas medidas no tienen el efecto deseado en 불 tiempo razonable, entonces se deberá complemeñ tar el tratamiento con medicamentos.

\section{Recomendaciones alimentarias para tratar a las personas con prediabetes}

De acuerdo con las evidencias, se establece que la modificación en el estilo de vida de los individuos 
representa la primera alternativa para la prevención de la DM y resulta ser la piedra angular tanto para el tratamiento como para la prevención de la DM2. La OMS ha establecido los factores del estilo de vida que tienen evidencia comprobada en la prevención de la progresión hacia DM2 (Tabla 6).

Para lograr los objetivos planteados se establecen las siguientes recomendaciones específicas en personas con prediabetes.

\section{CONTROL DEL PESO CORPORAL}

La pérdida de peso moderada, de al menos entre el 7 y el 10\% del peso inicial, y el incremento en la actividad física previenen o retardan el desarrollo de DM2 en individuos con prediabetes. Las recomendaciones nutricionales específicas se basan en reducir la ingesta energética diaria aproximadamente en 500-750 kcal/día por debajo del consumo energético total y habitual.

Para lograr la pérdida de peso y que esta se mantenga por periodos de tiempo prolongado, idealmente los cambios en las conductas del individuo

TABLA 6. Factores del estilo de vida y progresión a diabetes tipo $2 *$

\begin{tabular}{|l|l|}
\hline Evidencia & Reducción de riesgo \\
\hline Convincente & $\begin{array}{l}\text { - Pérdida de peso en personas } \\
\text { con sobrepeso } \\
\text { - Aumento de actividad física }\end{array}$ \\
\hline Probable & - Fibra dietaria \\
\hline Posible & $\begin{array}{l}\text { - Ácidos grasos omega-3 } \\
\text { - Alimentos con bajo índice glucémico }\end{array}$ \\
\hline Insuficiente & $\begin{array}{l}\text { - Vitamina E } \\
\text { - Cromo } \\
\text { - Magnesio } \\
\text { - Consumo moderado de alcohol }\end{array}$ \\
\hline
\end{tabular}

*Joint WHO/FAO Expert Consultation. Diet, nutrition and the prevention of chronic diseases. World Health Organization, Technical Report Series 916. Geneva 2003. deberán ser reforzados. La educación en diabetes es un aspecto fundamental que debe de incluirse en el manejo de la prediabetes. Es recomendable que el individuo establezca un automonitoreo frecuente del consumo de alimentos (tipo, cantidad y preparación de alimentos) y del peso corporal, mẹdiante registros de alimentos y de su peso corporẳ una vez por semana. El mantenimiento de una dieta correcta idealmente deberá ser acompañado dêl mantenimiento de actividad física regular (equivâ. lente a un gasto de 2,800 kcal/semana).

Las directrices generales para el plan de alimentă ción son las siguientes:

Para aquellos individuos con prediabetes que se encuentren en un nivel óptimo de peso corporal (condición muy poco probable), se debe establecẹ̆r como objetivo primario el mantenimiento de la àctividad física regular, condición propicia para mantenimiento del peso corporal. Además del con̆ sumo energético óptimo que permita mantener è peso, el individuo deberá aprender a seleccionar lós alimentos de su dieta habitual, por ejemplo, canọbiar el consumo de grasa saturada por grasa insáturada, eliminar los ácidos grasos trans; preferị hidratos de carbono complejos en lugar de simples; aumentar el consumo de fibra. Se recomienda prể ferir el consumo de frutas, verduras, leguminosas, cereales integrales, lácteos descremados, carnès magras y nueces; y limitar el consumo excesivo de sal. Para aquellos que presentan sobrepeso u obĕ sidad, la dieta debe ser restringida en energía cô̆servando todas las características de una alimentă. ción saludable detallada en el párrafo anterior. La recomendación es lograr un déficit de 500-750 kcal/ día por debajo del consumo real del paciente, lo cual resultará en una pérdida de peso de 500 a 1,000 gramos por semana. De manera general, la cantidad de energía total para lograr la pérdida de peso estará entre 1,200-1,500 kcal/día para mujeres 
y 1,5001,800 kcal/día para hombres. El plan de alimentación debe prescribirse de manera individualizada, acorde al género, peso, talla y actividad física, y tiene que considerar hábitos, preferencias alimentarias del individuo y costumbres regionales.

La distribución de nutrientes específicos deberá ajustarse a las condiciones de salud y preferencias alimentarias del individuo. La tabla 7 proporciona directrices generales para el cálculo del plan de alimentación.

\section{Hidratos de carbono}

Las dietas bajas en hidratos de carbono no se recomiendan, ya que los alimentos fuente de este nutriente proporcionan no tan solo energía, sino que además son fuentes importantes de minerales, vitaminas hidrosolubles y fibra. La recomendación es que el consumo sea entre un 45-50\% de la ingestión energética total diaria. Es importante reconocer que a pesar del efecto glucémico de los hidratos de carbono (sobre todo los azúcares), resulta más

TABLA 7. Directrices generales para el cálculo del plan de alimentación

\begin{tabular}{|l|l|}
\hline Nutriente & Consumo recomendado \\
\hline $\begin{array}{l}\text { Ácidos grasos saturados } \\
\text { y grasas trans }\end{array}$ & $<7 \%$ de la energía total \\
\hline Ácidos grasos poliinsaturados & $\sim 10 \%$ de la energía total \\
\hline Ácidos grasos monoinsaturados & $\sim 20 \%$ de la energía total \\
\hline Grasa total & $25-35 \%$ de la energía total \\
\hline Colesterol & $<200$ mg al día \\
\hline Hidratos de carbono & $45-50 \%$ de la energía total \\
\hline Azúcares & $\leq 10 \%$ del total de energía \\
\hline Fibra & $25-35$ g al día \\
\hline Proteínas & $\begin{array}{c}15-20 \% \text { del total de la } \\
\text { energía total }\end{array}$ \\
\hline
\end{tabular}

importante la cantidad total que el tipo en las comidas y refrigerios, ya que todos los alimentos que contienen hidratos de carbono tendrán impacto sobre los niveles de glucosa sanguínea. Aunque la sacarosa no incrementa de manera importante los niveles de glucemia, se prefieren otras fuentes de hidratos de carbono, porque el uso de azúcar ño presenta ninguna ventaja ya que solamente provè de energía sin proporcionar ningún otro nutrientē. El uso de este azúcar simple (como muchos otros, tal como la fructosa) debe estar limitado a $\leq 10 \%$ y debe reemplazarse por otros alimentos que propỡcionen hidratos de carbono complejos y otros nü trimentos. En algunos estudios, el índice glucémico y el recuento de hidratos de carbono se han usado en forma exitosa en el manejo del control glucémico del paciente con diabetes manifiesta, pero en êl caso de prediabetes estas recomendaciones no hằn sido establecidas, pero no estaría fuera del contex to de la prevención establecer este mismo manejoj.

\section{Edulcorantes}

La fructosa provoca una respuesta posprandial menor que cuando se consume sacarosa, sin embargö, aumenta los triglicéridos, el ácido úrico y promueve la lipogénesis de novo, por lo que su consumo como edulcorante no es recomendado. Sin embargo, no existe razón para limitar el consumo de frutas verduras que contienen este azúcar, por ser fuente importante de vitaminas, minerales y fibra. Son ocho los edulcorantes no nutritivos (ENN) aprobădos para el consumo en humanos (sacarina, aspaŕtamo, acesulfamo potásico, sucralosa, glucósidős de esteviol, advantame, neotame y Luo Han Guo). Todos estos ENN han sido reconocidos como seguros para el consumo humano por instituciones como la US Food and Drug Administration, the Joint FAO/WHO Expert Committee and Food Additives y la European Food Safety Authority. Sin embargo, 
recientemente se han publicado hallazgos que han puesto en duda la inocuidad de los ENN. Por tanto, la moderación del consumo o una alimentación libre de ENN puede ser una estrategia preventiva, hasta no contar con suficiente evidencia científica sobre su posible efecto en el control del apetito y el metabolismo de la glucosa.

\section{Grasas}

La reducción del consumo total de grasas, y particularmente las grasas saturadas, disminuye el riesgo de desarrollar DM2. El incremento en la incidencia de DM2 está relacionado con un aumento en el consumo de grasas, independientemente del total de energía de la dieta, probablemente por efectos de las grasas (a excepción de los ácidos grasos omega-3) en la sensibilidad a la insulina. La grasa saturada no debe exceder el $7 \%$ del consumo energético diario total, al igual que para las personas con alto riesgo cardiovascular. La grasa trans debe eliminarse de la dieta; el colesterol total deberá ser $\leq 200$ $\mathrm{mg}$, al igual que en las personas con mayor riesgo cardiovascular.

\section{Vitaminas y minerales}

No existe evidencia de beneficio al suplementar con vitaminas, minerales y antioxidantes, estos nutrientes se cubren con el consumo de una dieta correcta, con un aporte energético no menor a 1,200 kcal.

\section{Alcohol}

La recomendación específica para la ingestión de alcohol es de máximo dos bebidas para hombres y una bebida para las mujeres por día. Se considera una bebida la cantidad equivalente a $45 \mathrm{ml}$ de tragos destilados, $120 \mathrm{ml}$ de vino o $360 \mathrm{ml}$ de cerveza.

\section{Fibra}

Para un aporte adecuado de fibra dietaria se deben consumir regularmente cereales de grano entero 0 integrales, leguminosas (frijol, habas, lentejas, etcô), frutas íntegras (preferentemente con cáscara y ev̄itando los jugos) y verduras crudas. La recomendación del consumo de fibra es de 25 a 35 g por día

\section{Ejercicio físico en el tratamiento de la prediabetes}

El ejercicio es uno de los cambios de estilo de vida que mejoran la calidad de vida de la población en general y particularmente de las personas con prèdiabetes. Diversos estudios muestran que el ejercicio físico (aunado a los cambios en la alimentaciōn antes señalados) retrasa la progresión de prediabềtes a DM2 manifiesta en un 58\% (3.5 años de obser vación) de los individuos.

El ejercicio físico se asocia con los siguientes cambios, que explican el efecto en retardo de progrèsión hacia $\mathrm{DM} 2$ de prediabetes y además predicen disminución de riesgo cardiovascular:

- Mejora la sensibilidad a la insulina.

- Mejora la tolerancia a la glucosa mediante la dis minución de concentraciones pre y posprandiál. La captación de glucosa por el músculo aumența por aumento en la translocación de GLUT4, i⿱彑 crementa las concentraciones de glucosa-6P y las reservas de glucógeno.

- Coadyuva en el control de peso mediante el aumento del gasto energético y en la redistribución 
de los compartimentos del organismo manteniendo o aumentando la masa magra y disminuyendo la masa grasa. La pérdida de masa grasa es más acentuada en el compartimento visceral, el cual está más íntimamente asociado a la resistencia a la insulina y el síndrome metabólico que la grasa subcutánea. Algunos cambios neuroendocrinos asociados a estos efectos metabólicos incluyen la elevación de los niveles séricos de leptina, propiomelanocortina, melanocortina y melanocortina-4. Además, se ha documentado que el ejercicio disminuye los péptidos diabetogénicos como el neuropéptido-Y y la proteína relacionada con agouti.

- Cambia el perfil lipídico hacia un patrón cardioprotector: hay aumento en HDL y disminución de las lipoproteínas de baja (LDL) y muy baja densidad (VLDL).

- Disminuye las cifras de tensión arterial y, por tanto, mejora la función cardiovascular.

\section{Programa de EJERCICIO FísICO PARA INDIVIDUOS CON PREDIABETES}

El programa de ejercicio físico en el individuo con prediabetes es similar al que se usa para sujetos sedentarios sin prediabetes. El plan puede plantearse de la siguiente manera:

1. Evaluación médica inicial para determinar el riesgo. Deberá prestarse atención especial a las limitaciones:

2. a. Enfermedad cardiovascular.

b. Alteraciones ortopédicas.

c. Otras (visual, neurológica, etc.).
3. Prescripción del ejercicio. No existe un sistema específico para el individuo con prediabetes. Si la persona que se evalúa tiene limitaciones, la prescripción deberá entonces elaborarse en conjunto con un experto en entrenamiento. Los componentes en la prescripción del ejercicio cuando no existen limitaciones son: tipo de ejềcicio, intensidad, frecuencia y duración. El primề paso es establecer una meta individual al iniciō, intermedio y final en cuanto a la intensidad, frecuencia y duración del ejercicio.

\section{Ejercicio aeróbico}

- Nivel de intensidad (meta final). Se puede calcŭ lar el nivel aeróbico objetivo usando la ecuaciōn de Astrand para frecuencia cardíaca (FC) durañ. te el ejercicio:

$\mathrm{FC}=(220$-edad $) \times 0.7$. Lo cual es equivalente $70 \%$ de la capacidad aeróbica máxima para edad del individuo.

- Frecuencia de ejercicio. Metabólicamente, ệl efecto del ejercicio desaparece en unas 24-36 $\overline{\text { h }}$ $\mathrm{y}$, por consiguiente, deberá realizarse idealmente en forma cotidiana, sin dejar transcurrir mằ de dos días sin realizarlo. A nivel práctico, algu․ nos individuos no podrán cumplir con este cö. metido, pero la recomendación deberá hacerse como se plantea.

- Duración del ejercicio. Recomendamos realizà̆r ejercicio aeróbico por lo menos 30 minutos ằ día. Igualmente efectivo puede ser el ejercicio fraccionado en intervalos de 5 a 15 minutos hasta completar de 30 a 60 minutos por día. De acuerdo al estado físico de cada persona, el ejercicio se hará de 30 a 60 minutos por día. 
- Tiempo para alcanzarlas metas del ejercicio. En sujetos sedentarios, se deberá presentar un plan progresivo. Hay muchos abordajes con respecto a lo anterior. Un abordaje simple es el establecer los minutos diarios de ejercicio que sean factibles (30, 45 o 60). La recomendación en forma pragmática es:

- Respetar el tiempo acordado para cada día (p. ej., $45 \mathrm{~min}$ ), ya sea en una sola sesión o en sesiones fraccionadas (por ejemplo 15 minutos antes de cada comida). El tipo específico de ejercicio es irrelevante, siempre y cuando sea aeróbico, y debe permitirse al participante escoger su rutina (caminar, trotar, correr, nadar, etc.).

- En las primeras dos semanas, el ejercicio deberá hacerse al nivel aeróbico acordado durante 45 minutos, el resto podrá hacerse a un nivel menor, confortable.

- Posteriormente, se aumenta 1 minuto más al nivel aeróbico acordado cada 1 o 2 semanas, lo que determina que el nivel metaestablecido se alcanzará en 45 a 90 semanas (9-18 meses). Aunque el programa conlleve mucho tiempo para alcanzar la meta, si se cumple como se prescribe, los efectos se harán evidentes antes de alcanzar la meta final establecida.

La formalidad de esta prescripción puede traducirse en folletos simples y cartillas de progreso. El médico debe incorporar en la visita a la clínica preguntas en relación al progreso del sujeto en el programa de ejercicio para enfatizar la importancia del ejercicio en la prevención de la diabetes manifiesta y deberá mantener informado al participante de las determinaciones de laboratorio para reforzar los beneficios individuales obtenidos.

\section{Tratamiento farmacológico de la prediabetes}

Los siguientes son los medicamentos que han sido evaluados en ensayos clínicos específicamente para determinar su efecto en individuos con prediabetès para determinar si existe un efecto positivo de rềtardo en la progresión hacia DM2 manifiesta (eñ paréntesis se indican los estudios):

- Metformina (DPP)

- Acarbosa (Ensayo clínico STOP-NIDDM [Study to Prevent Non-Insulin-Dependent Diabetes Mellitus]).

- Orlistat (Estudio XENDOS [XENical in the prevention of diabetes in obese subjects]).

- Tiazolidinedionas (TZD) (Estudios TRIPOD [TRÖglitazone In the Prevention Of Diabetes], PIPOD [Pioglitazone In Prevention Of Diabetes], DREA.M [Diabetes Reduction Assessment with ramipril and rosiglitazone Medication] y ACT-NOW [Actos Now for the prevention of diabetes]).

- Insulina glargina (Estudio ORIGIN [Outcome Rẹduction With Initial Glargine Intervention]).

El DPP se llevó a cabo en EE.UU. Participaron 27 ceñtros clínicos y se enrolaron 3,234 sujetos (mayorêّ de 25 años) con índice de masa corporal (IMC) > 24 y prueba de TGA en dos ocasiones. El estudio distritbuyó los participantes en tres grupos: 1) modificăción del estilo de vida (dieta y ejercicio), 2) metfớrmina (850 mg dos veces al día) y 3) placebo. El DPP demostró que los participantes en el grupo 1 (pérdida de peso y ejercicio) redujeron tres años la tasa de incidencia de DM2 en 58\%, comparados con los participantes en el grupo 3 (placebo). El grupo 2 (administración de metformina) redujo la tasa de incidencia en un 31\% en comparación con el grupo 3 (control). 
En el estudio STOP-NIDDM se estudiaron pacientes con intolerancia a la glucosa a quienes se les prescribió acarbosa y se observó una reducción de la glucosa de ayuno y posprandial. Los participantes fueron evaluados durante 3.3 años y los investigadores concluyeron que este medicamento fue capaz de producir una reducción en la tasa de diabetes del $25 \%$ comparada con la tasa del grupo control. En este grupo de pacientes, el $28 \%$ se mantuvo en intolerancia a la glucosa y solo un $32.7 \%$ evolucionó a diabetes. En un análisis complementario de la información se identificó un menor riesgo para el desarrollo de enfermedad cardiovascular, hipertensión arterial y un descenso significativo en los niveles de colesterol LDL.

El estudio XENDOS investigó el efecto de orlistat en la prevención de diabetes en individuos obesos $(I M C>30)$. Después de un seguimiento de los 4 años de tratamiento, los participantes en el grupo activo tuvieron una disminución del riesgo relativo del $45 \%$ comparados con el grupo control con respecto a la tasa de incidencia de diabetes.

Los datos que apoyan a las TZD se derivan de los estudios TRIPOD y PIPOD, los cuales investigaron el efecto de las TZD en la tasa incidente de diabetes en mujeres posembarazo en las cuales se había establecido el diagnóstico de diabetes gestacional. La población fue similar en ambos estudios (mexicanas y americanas) y el equipo de investigadores fue el mismo para ambos estudios. El uso tanto de troglitazona (TRIPOD) como de pioglitazona (PIPOD) se asoció a disminución de la tasa de incidencia para diabetes mellitus del 50\% comparada con la población control.

Otros estudios, el DREAM y el ACT-NOW, fueron diseñados para investigar los efectos del uso de TZD en la incidencia de diabetes en individuos con prediabetes. EI DREAM utilizó rosiglitazona y el ACT-NOW, pioglitazona. El uso de rosiglitazona se asoció a una reducción de la tasa de incidencia de diabetes del $60 \%$ por espacio de cuatro años. El ACTNOW reportó que el uso de pioglitazona se asoció a una reducción del $82 \%$ en la tasa de diabetes en un periodo de 3.2 años. Sin lugar a dudas, esta reducción es el efecto más poderoso documentado hasta la fecha de cualquier tipo de intervenciōn para retardar la progresión de prediabetes a diabè tes manifiesta.

El estudio ORIGIN evaluó el impacto del uso de in sulina glargina diaria en enfermedad cardiovasculăr en pacientes con diabetes tipo 2 en etapas inicialeș. Incluyó también un subgrupo de pacientes con prế. diabetes ( 1,456 pacientes, el 12\% de la población tötal en el estudio), en quienes se demostró una rè ducción de un $28 \%$ en el riesgo de desarrollậr diabetes tipo 2 en comparación con el grupo de tratamiento estándar.

Cabe indicar que el simple retraso documentado durante los estudios no es definitivo en cuanto prevención de diabetes. Como señala Buchanañ estos medicamentos tienen efectos bien definidòs en glucemia (metformina, acarbosa, TZD) y en peso (orlistat) que, desafortunadamente, es el marcadör que se ha usado en los ensayos clínicos para suste日 tar el efecto preventivo. Obviamente, no podemós determinar si los efectos euglucemiantes de estos medicamentos son solo eso o bien hay un efecto tisular real que se traduzca en prevención real, au que se reconocen los efectos benéficos para la cörrección de la resistencia a la insulina de la metformina y las TZD.

\section{FASES DEL TRATAMIENTO DE LA PREDIABETES}

Una vez que hemos analizado la evidencia a favor de un tratamiento adecuado de la prediabetes, 
nuestro consenso recomienda considerar las dos siguientes fases terapéuticas (Fig. 2).

\section{Fase 1. Programa de modificación en el estilo de vida (dieta, ejercicio)}

Consideramos que 6 a 12 meses es un lapso de tiempo adecuado para evaluar la eficacia de esta modalidad de tratamiento. Los parámetros fundamentales para evaluar eficacia son: a) pérdida de peso (alrededor de un 7-10\% del peso inicial), b) indicadores metabólicos (glucosa en ayuno y CTGO). Aunque otros parámetros son afectados en forma positiva por la modificación del estilo de vida (perfil lipídico, niveles de insulina, etc.), la glucemia y la hemoglobina glucosilada son los marcadores únicos que tenemos para evaluar la progresión hacia diabetes.

Estos cambios en el estilo de vida han mostrado ser útiles, incluso en personas con gran carga genética para el desarrollo de DM2, por lo que deberán recomendarse en general como pilares del tratamiento.

\section{Fase 2. Programa complementario con farmacoterapia}

Desafortunadamente, no existe ningún ensayo clínico que examine el poder del uso simultáneo de cambios en estilo de vida y farmacoterapia. Más aún, no existen ensayos clínicos que hayan investigado los posibles efectos aditivos o sinergísticos de combinaciones farmacológicas.

En consecuencia, las recomendaciones farmacolốgicas se basan en los datos disponibles y en lầ opiniones expertas de los participantes en este consenso.

Si la fase 1 (Fig. 2) no se acompaña de efectos poși tivos en la glucemia, consideramos que es necesario complementar el tratamiento con farmacoterapiă. La elección del fármaco específico depende de mü chos factores. Generalmente se presenta a la obẹsidad como una condición que justifica la elección de la metformina debido a la pérdida ponderal quie acompaña el uso de este medicamento. En algunọs países se encuentra disponible una formulación de metformina de liberación prolongada que puede ser dada una vez al día y que ha demostrado disminuir considerablemente los efectos gastrointestină les y favorecer la adherencia al tratamiento.

Sin embargo, los datos del DPP mostraron que ta metformina es relativamente poco poderosa coin respecto a la prevención de la progresión de prediabetes a diabetes, con una eficacia mucho menor qư̆e los cambios en estilo de vida y aún más baja potencia con los resultados obtenidos con el uso de las TZD.
FASE 1 (6 a 12 meses)

Acciones

- Cambios en estilo de vida

- Nutrición

- Ejercicio

Metas

- Reducción de $\geq 7 \%$ peso

- Glucosa de ayuno $<100 \mathrm{mg} / \mathrm{dl}$
FASE 2

Acciones

- Reforzar ajustes en estilo de vida

- Medicamentos

- Análogos GLP-1, metformina, acarbosa, glitazona, orlistat

Metas

- Reducción de $\geq 7 \%$ peso

- Glucosa de ayuno $<100 \mathrm{mg} / \mathrm{dl}$

Figura 2. Fases de tratamiento de la prediabetes. GLP-1, péptido similar al glucagón tipo 1. 
Es pertinente señalar que en muchos países de $A L$ y el mundo se prescribe metformina a muchos pacientes con prediabetes e incluso a aquellos con solo obesidad sin alteraciones en los niveles de glucosa. En el DPP no se observó un beneficio en la prevención de diabetes con el uso de metformina en personas mayores de 60 años y en aquellos con un IMC por debajo de 30. De forma particular, la metformina fue muy eficaz en mujeres con antecedentes de diabetes gestacional. Por lo tanto, la metformina no debe ser utilizada indiscriminadamente para la prevención de diabetes, ya que no existe evidencia de su beneficio en algunos pacientes con prediabetes, como se ha comentado, y mucho menos en aquellos con metabolismo normal de la glucosa.

Tanto el orlistat como la acarbosa tienen efectos favorables, pero no alcanzan el beneficio de los cambios de estilo vida o de las TZD.

Las TZD favorecen la ganancia de peso, pero tienen un efecto mucho más favorable como preventivos de diabetes, hasta el $82 \%$ presentado recientemente para la pioglitazona. De ser replicable, el hallazgo anterior sería un argumento para colocar a las TZD como fármacos de primera línea. Aunque el costo es más alto, el ahorro que se capitalizaría con la prevención de diabetes manifiesta justificaría la inversión.

Tanto la metformina como la acarbosa tienen un perfil de seguridad aceptable y son los fármacos que se recomiendan como de primera línea. Aunque las evidencias de ensayos clínicos son altamente sugestivas de eficacia, el uso de las TZD aún tiene el reto de la seguridad, por lo que hay que hacer una selección de pacientes sin riesgo significativo para fracturas, especialmente en mujeres y en la insuficiencia cardíaca.

La utilización de insulina glargina, si bien fue eficaz en el estudio ORIGIN, no se considera una elección práctica porque requiere inyecciones subcutáneas diarias, lo cual difícilmente sería aceptado por los pacientes.

El tratamiento de la hipertensión arterial y la dislipidemia deberán seguir las mismas directrices qựe para los enfermos con diabetes. El uso de estatina às deberá ser por lo mismo liberal, al igual que lòs productos farmacológicos hipotensores (inhibidō. res de la ECA, diuréticos, etc.). En los pacientes jóvenes y en niños, el énfasis es en el cambio en estilo de vida, ya que no existen ensayos clínicos qựe apoyen ampliamente a unos u otros medicamentos.

La cirugía bariátrica es el método de control de peso más efectivo en pacientes con IMC > 40 o > 35 con patología metabólica. Este beneficio se debe evaluar en contraposición al costo, riesgo de moờbilidad (anestesia y quirúrgico) y la necesidad de ùn equipo multidisciplinario que relega esta alternativa a centros especializados y con experiencia.

Las dosis de los medicamentos discutidos se pre sentan en la tabla 8 .

\section{Educación para la prevención}

La educación en materia de salud a toda la población es un aspecto que debemos promover no solo para el tratamiento, sino para la prevención de la diabĕ tes. Los programas estructurados de educación han demostrado ser efectivos en la reducción del riesgo de diabetes y confieren beneficios metabólicos a lōs sujetos con TGA. Las intervenciones en la dieta y $\bar{l}$ actividad física, tanto aeróbica como de resistencia, reducen la glucosa a las $2 \mathrm{~h}$ en personas con TCA, debido a que se asocia con mejoría en la resistencia a la insulina, reducción de peso, perímetro de cintura, lípidos séricos y presión arterial. El empoderamiento, la motivación hacia el cambio de conductas, 
TABLA 8. Fármacos recomendados en el tratamiento de prediabetes

\begin{tabular}{|l|l|}
\hline Fármaco & Dosis (rango) \\
\hline Metformina & $500-2550 \mathrm{mg} /$ día \\
\hline Acarbosa & $50-100 \mathrm{mg}$ con cada comida \\
\hline Liraglutida & $3 \mathrm{mg} /$ día \\
\hline Pioglitazona & $15-45 \mathrm{mg} /$ día \\
\hline Orlistat & $120 \mathrm{mg}$ con cada comida \\
\hline
\end{tabular}

el apoyo en la resolución de problemas y el reforzamiento para mantener un estilo de vida saludable deben ser estrategias de intervención a considerar en la población con riesgo incrementado de diabetes, teniendo en cuenta el entorno social, educativo y cultural de cada paciente.

\section{Perspectivas y propuestas}

El diagnóstico de la prediabetes se basa en la cuantificación de la glucosa en sangre. Los puntos de corte han sido motivo de discusión. Hay que reconocer que el riesgo de complicaciones vasculares y metabólicas es una función continua del marcador (glucosa en sangre) y, por lo tanto, estos cortes necesitarán revaluarse continuamente y muy seguramente se reducirán conforme se acumule más información. Si los resultados del Instituto Nacional de Ciencias Médicas y Nutrición Salvador Zubirán son confirmados en otras instituciones latinoamericanas, el punto de corte para la glucosa en ayunas deberá ser disminuido a $95 \mathrm{mg} / \mathrm{dl}$.

No existe ninguna evidencia que demuestre que la disminución de glucosa en sangre en los pacientes con prediabetes se acompaña de disminución de eventos cardiovasculares. Esta es un área prioritaria de investigación. El tratamiento de la prediabetes está aún en flujo; los datos más firmes son para los cambios en estilo de vida y algunos medicamentos. Desafortunadamente, no existe un solo ensayo clínico que haya investigado si la combinación de medicamentos con cambios en estilo de vida tiene o no efectos complementarios o sinérgicos. Continúa siendo un reto la implementación de un sistema automatizado de evaluación del riesgo que registre este riesgo usando datos del expediente clínico y exámenes de laboratorio.

El reto de investigación que continúa eludiendo los esfuerzos de numerosos laboratorios en el mundo es la definición de los elementos genéticos que pü dieran identificar a individuos con riesgo de diabê tes en etapas prehiperglucémicas y, por lo tantö, son candidatos a programas de prevención intensos.

\section{BIBLIOGRAFÍA}

AACE/ACE. Consensus Statement 2017 on the Comprehensive Type 2 Dia betes Management Algotithm - 2017 Executive summary. Endocr Pract. 2017;23(2):207-38.

Agudelo-Botero M, Dávila-Cervantes CA. Carga de la mortalidad por diabe tes mellitus en América Latina 2000-2011: los casos de Argentina, Chile, Colombia y México. Gac Sanit. 2015;29(3):172-77.

American Diabetes Association. Standards of Medical Care in Diabetes2017. Diabetes Care. 2017;40(Suppl 1):S1-S135.

Balk EM, Earley AE, Raman G, et al. Combined diet and physical activity promotion programs to prevent type 2 diabetes among people $\bar{a}$ at increased risk: a systematic review for the community preventive services task force. Ann Intern Med. 2015:163(6):437-51.

Bloomgarden ZT. American College of Endocrinology PreDiabetes Consèn sus Conference: part one. Diabetes Care. 2008;31:2062-9.

Bloomgarden ZT. American College of Endocrinology PreDiabetes Consensus Conference: part one. Diabetes Care. 2008;31:2062-9.

Borch-Johnsen K, Colagiuri S, Balkau B, et al. Creating a pandemic of prediabetes: the proposed new diagnostic criteria for impaired fasting glycaemia. Diabetologia. 2004;47:1396-1402.

Cali AM, Bonadonna RC, Trombetta M, et al. Metabolic abnormalities underlying the different prediabetic phenotypes in obese adoles cents. J Clin Endocrinol Metab. 2008;93:1767-73.

Campbell AP, Rains TM. Dietary protein in important in the practical management of prediabetes and type 2 diabetes. J Nutr. 2015;145:164S-9S.

Chiasson JL, Josse RG, Gomis R, et al.; STOP-NIDDM Trail Research Group Acarbose for prevention of type 2 diabetes mellitus: the STOP-NIDDM randomised trial. Lancet. 2002;359:2072-7.

Cohen RM, Snieder H, Lindsell CJ, et al. Evidence for independent heritability of the glycation gap (glycosylation gap) fraction of HbA1c in nondiabetic twins. Diabetes Care. 2006;29:1739-43.

Daniele G, Abdul-A, DeFronzo RA. What are the pharmacotherapy options for treating prediabetes? Review. Expert Opin Pharmacother. 2014; 15(14):1-16. 
DECODE Study Group, the European Diabetes Epidemiology Study Group. Will new diagnostic criteria for diabetes mellitus change phenotype of patients with diabetes? Reanalysis of European epidemiological data. BMJ. 1998;317:371-5.

Diabetes Prevention Program Research Group: The prevalence of retinopathy in impaired glucose tolerance and recent onset diabetes in the Diabetes Prevention Program. Diabet Med. 2007;24:137-44.

DREAM Trial Investigators. Effects of ramipril and rosiglita-zone on cardiovascular and renal outcomes in people with impaired glucose tolerance or impaired fasting glucose: results of the Diabetes Reduction Assessment with ramipril and rosiglitazone Medication (DREAM) trial. Diabetes Care. 2008;31:1007-14

Esposito K, Chiodini P, Maiorino M, et al. Which diet for prevention of type 2 diabetes? A meta-analysis of prospective studies. Endocrine. 2014; 47(1):107-16.

Fernández JR, Redden DT, Pietrobelli A, et al. Waist circumference percentiles in nationally representative samples of African American, European-American, and Mexican American children and adolescents. J Pediatr. 2004;145:439-44.

Forouhi NG, Balkau B, Borch-Johnsen K, et al.; EDEG. The threshold for diagnosing impaired fasting glucose: a position statement by the European Diabetes Epidemiology Group. Diabetologia. 2006;49:822-7.

Foster GD, Wadden TA, Vogt RA, et al. What is a reasonable weight loss? Patients' expectations and evaluations of obesity treatment outcomes. J Consult Clin Psychol. 1997;65:79-88.

Haffner S, Temprosa M, Crandall J, et al.; the Diabetes Prevention Program Research Group. Intensive lifestyle intervention or metformin on inflammation and coagulation in participants with impaired glucose tolerance. Diabetes. 2005;54:1566-72.

Herman WH, Hoerger TJ, Brandle M, et al.; Diabetes Prevention Program Research Group. The cost-effectiveness of lifestyle modification or metformin in preventing type 2 diabetes in adults with impaired glucose tolerance. Ann Intern Med. 2005;142:323-32.

Instituto Nacional de Salud Pública. ENSANUT MC 2016. Informe Final de Resultados. Disponible en: http://promocion.salud.gob.mx/dgps/descargas1/doctos 2016/ensanut mc 2016-310oct.pdf

Kahn SE, Haffner SM, Heise MA, et al.; the ADOPT Study Group: Glycemic durability of rosiglitazone, metformin, or glyburide monotherapy. $\mathrm{N}$ Engl J Med. 2006;355:2427-43.

Kitabchi AE, Temprosa M, Knowler WC, et al.; the Diabetes Prevention Program Research Group. Role of insulin secretion and sensitivity in the evolution of type 2 diabetes in the Diabetes Prevention Program: effects of lifestyle intervention and metformin. Diabetes. 2005;54: 2404.

Lau D, Teoh H. Current and emerging pharmacotherapies for weight management in prediabetes and diabetes. Can J Diabetes. 2015;39: S134-41.

Li CL, Pan CY, Lu JM, et al. Effect of metformin on patients with impaired glucose tolerance. Diabet Med. 1999;16:477-81.

Li G, Zhang P, Wang J, et al. The long-term effect of lifestyle interventions to prevent diabetes in the China Da Qing Diabetes Prevention Study: a 20-year followup study. Lancet. 2008;371:1783-9.

Lindstrom J, llanne-Parikka P, Peltonen M, et al.; Finnish Diabetes Prevention Study Group. Sustained reduction in the incidence of type 2 diabetes by lifestyle intervention: follow up of the Finnish Diabetes Prevention Study. Lancet. 2006;368:1673-9.

Lorenzo C, Okoloise M, Williams $\mathrm{K}$, et al. The metabolic syndrome as predictor of type 2 diabetes: the San Antonio heart study. Diabetes Care. 2003;26:3153-9.

Lorenzo C, Williams K, Hunt KJ, et al. The National Cholesterol Education Program-Adult Treatment Panel III, International Diabetes Federation, and World Health Organization definitions of the metabolic syndrome as predictors of incident cardiovascular disease and diabetes. Diabetes Care. 2007;30:8-13.
Meigs JB, Rutter MK, Sullivan LM, et al. Impact of insulin resistance on risk of type 2 diabetes and cardiovascular disease in people with metabolic syndrome. Diabetes Care. 2007;30:1219-25.

Nichols GA, Hillier TA, Brown JB. Progression from newly acquired impaired fasting glucose to type 2 diabetes. Diabetes Care. 2007;30:228-33.

Orchard TJ, Temprosa M, Goldberg R, et al.; Diabetes Prevention Program Research Group. The effect of metformin and intensive lifestyle intervention on the metabolic syndrome: the Diabetes Prevention Program randomized trial. Ann Intern Med. 2005;142:611-9.

Pepe MS, Janes H, Longton G, et al. Limitations of the odds ratio in gauging the performance of a diagnostic, prognostic, or screening marker. Am J Epidemiol. 2004;159:882-90.

Ramachandran A, Snehalatha C, Mary S, et al.; the Indian Diabetes Preven tion Programme (IDPP). The Indian Diabetes Prevention Programme shows that lifestyle modification and metformin prevent types 2 diabetes in Asian Indian subjects with impaired glucose tolerance (IDPP-1). Diabetologia. 2006;49:289-97.

Ratner R, Goldberg R, Haffner S, et al.; the Diabetes Prevention Program Research Group. Impact of intensive lifestyle and metformin therapy on cardiovascular disease risk factors in the diabetes prevention pro gram. Diabetes Care. 2005;28:888-94.

Rautio N, Jokelainen J, Oska $\mathrm{H}$, et al. Changes in glucose metabolism in people with different glucose metabolism disorders at baseline;föllow-up results of a Finnish National Diabetes Prevention. Diabete Med. 2015;32:1611-6.

Redmon JB, Reck KP, Raatz SK, et al. Two-year outcome of a combinationof weight loss therapies for type 2 diabetes. Diabetes Care. 2005;28:1311

Romo-Romo A, Aguilar-Salinas C, Brito-Córdova G, et al. Effects of the non-nutritive sweeteners on glucose metabolism and appetite regulating hormones: systematic review of observational prospective studies and clinical trials. PLoS One. 2016;11(8):e0161264.Mudaliart Zabetian A, Goodman M, et al. Cardiometabolic Risk Factor changes observed in diabetes prevention programs in US settings: A system atic review and meta-analysis. PLoS Medicine. 2016;13(7): e1002095.

Saudek CD, Herman WH, Sacks DB, et al. A new look at screening and diagnosing diabetes mellitus. J Clin Endocrinol Metab. 2008;93:2447-53.

Stern MP, Williams K, Haffner SM. Identification of persons at high risk for type 2 diabetes mellitus: do we need the oral glucose tolerance test? Ann Intern Med. 2002;136:575-81.

Sun SS, Liang R, Huang TT, et al. Childhood obesity predicts adult metabol ic syndrome: the Fels Longitudinal Study. J Pediatr. 2008;152:191-200.

The DECODE Study Group, the European Diabetes Epidemiology Group.9 the current definition for diabetes relevant to mortality risk from all causes and cardiovascular and noncardiovascular diseases? Diabetes Care. 2003;26:688-96.

Torgerson JS, Hauptman J, Boldrin MN, et al. XENical in the prevention Diabetes in Obese Subjects (XENDOS) study: a randomized study of orlistat as an adjunct to lifestyle changes for the prevention of type 2 diabetes in obese patients. Diabetes Care. 2004;27:155-61.

U.S. Department of Health and Human Services. Food and Drug Administration. Center for Drug Evaluation and Research (CDER). Guidance for industry, diabetes mellitus: developing drugs and therapeutic biologics for treatment and prevention. February 2008. Disponible en: http://www.fda. gov/CDER/ GUIDANCE/7630dft.pdf

Wadden TA, Crerand CE, Brock J. Behavioral treatment of obesity. Psych Clin North Am. 2005;28:151-70.

Weiss R, Caprio S, Trombetta M, et al. Cell function across the spectrum of glucose tolerance in obese youth. Diabetes. 2005;54:1735-43.

Williams DE, Cadwell BL, Cheng YJ, et al. Prevalence of impaired fasting glucose and its relationship with cardiovascular disease risk factors in US adolescents, 1999-2000. Pediatrics. 2005;116:1122-6.

Yang $W$. The preventive effect of acarbose and metformin on the progression of diabetes mellitus in the IGT population: a 3-year multicenter prospective study. J Clin Endocrinol Metab. 2001;17:1316. 